\title{
Ear Acupuncture for Acute Sore Throat: A Randomized Controlled Trial
}

\author{
David A. Moss, MD, and Paul Crawford, MD
}

Background: Sore throat is a common cause of pain in outpatient encounters. Battlefield auricular acupuncture (the placing of needles in specific points in the ear) is a modality used to treat acute pain associated with a variety of ailments. The aim of our study was to determine whether auricular acupuncture reduces pain, medication usage, and missed work hours when added to standard therapy in adult patients with acute sore throat.

Methods: We conducted an unblinded, pragmatic, randomized controlled trial among adult, nonpregnant patients presenting to an Air Force family medicine clinic with pain from acute sore throat. A total of 54 patients were followed for $\mathbf{4 8}$ hours after treatment.

Results: Patients receiving auricular acupuncture reported lower pain scores than those who did not at 15 minutes (6.0 [95\% confidence interval (CI), 5.4-6.6] vs 2.6 [95\% CI, 1.7-3.5]; $P<.0001), 6$ hours (4.8 [95\% CI, 4.0-5.6] vs 2.5 [95\% CI, 1.6-3.4]; $P=.0005)$, and 24 hours (4.1 [95\% CI, 3.34.9] vs 1.3 [95\% CI, 1.0-2.8]; $P=.0006)$. They also reported taking fewer cumulative doses of pain medication at 6 hours $(1.07$ [95\% CI, 0.69-1.45] vs 0.39 [95\% CI, 0.2-0.58]; $P=.003), 24$ hours (2.63 [95\% CI, 1.95-3.31] vs 1.37 [95\% CI, 0.92-1.82]; $P=.004)$, and 48 hours (4.07 [95\% CI, 2.95.24] vs 2.19 [95\% CI, 1.44-2.94]; $P=.009)$. There was no difference in time missed from work between the auricular acupuncture and standard therapy groups.

Conclusions: Compared with usual treatment, battlefield auricular acupuncture was associated with reduced sore throat pain for 24 hours and decreased use of pain medication for up to 48 hours. There was no apparent effect on hours missed from work. (J Am Board Fam Med 2015;28:697-705.)

Keywords: Acupuncture; Alternative Medicine; Clinical Trials, Randomized; NSAIDs; Pain Management; Sore Throat

Acute sore throat resulting from various upper respiratory infections is a common cause of doctor visits—15 million in 2009—and lost work. ${ }^{1}$ Regardless of its cause, symptomatic treatment with nonsteroidal anti-inflammatory drugs (NSAIDs) is often implemented despite whether antibiotics are appropriate. ${ }^{2,3}$ NSAIDs are associated with gastrointestinal bleeding, renal failure, and other serious adverse events. Thus, patients often use alternative remedies such as salt water gargles, throat spray,

This article was externally peer reviewed.

Submitted 8 January 2015; revised 27 May 2015; accepted 8 June 2015.

From the US Air Force, North Las Vegas, NV.

Funding: DAM and PC received a grant from the Air Force Medical Support Agency's Research and Development Division (AFMSA/SGRS) during the conduct of the study.

Conflict of interest: none declared.

Corresponding author: David A. Moss, MD, 4700 North Las Vegas Blvd, NAFFB, NV 89191 (E-mail: david.moss.3@us.af.mil). lozenges, or ice chips to alleviate their pain. ${ }^{4,5} \mathrm{Au}-$ ricular acupuncture is another modality that is reported to be effective at relieving many types of pain.

Auricular acupuncture (placing needles in various points in the ear) is a form of integrative medicine in which particular points on the ear, when stimulated, are believed to correspond with specific areas of the body. The purpose of auricular acupuncture, similar to traditional acupuncture, is to balance the flow of the body's energy, or qi. Mechanisms for its effectiveness are thought to relate to the inhibition of neurotransmitters and inflammatory markers such as substance $\mathrm{P}$ and interleukins, as well as the release of endogenous opioids. ${ }^{6,7}$ Studies using functional magnetic resonance imaging and positive electron tomography also show that specific areas of the brain relating to pain sensation are either stimulated or inhibited when auricular acupuncture needles are in place. ${ }^{8}$ 
Battlefield acupuncture (BFA) is a specific auricular acupuncture technique developed by Niemtzow $^{9}$ and refers to placing needles in 5 specific points on the ear (see Figure 1). This is done using semipermanent acupuncture needles in a rapid fashion for acute pain relief. As opposed to traditional auricular acupuncture, which can be complicated and requires extensive training, this technique uses a simple, systematic approach to placing the needles that can be learned by physicians in a 4-hour workshop. In addition, BFA takes only 3 to 10 minutes to administer to patients. Because the needles are semipermanent and very small, the needles stay in place after the patient leaves the encounter and are allowed to fall out on their own over 3 to 7 days. There are limited data regarding this specific technique, though auricular acupuncture itself has been practiced since the 1950s. ${ }^{10}$

Many physicians currently use BFA, and the technique is taught in multiple venues. ${ }^{11} \mathrm{BFA}$ has not been studied extensively, but there is a growing body of evidence supporting auricular acupuncture as an effective modality in relieving acute pain. This includes pain in the intra- and postoperative periods, ${ }^{12-14}$ low-back pain and pelvic pain associated with pregnancy, ${ }^{15}$ migraines,${ }^{16}$ and acute pain syndromes in an emergency department setting. ${ }^{17}$ Not all studies show positive results, ${ }^{18-20}$ but a recent meta-analysis showed an average mean difference in pain scores of -1.89 on a 10 -point visual analog scale. ${ }^{21}$

The purpose of this study is to evaluate the efficacy of BFA in addition to standard therapy compared with standard therapy alone in relieving pain from acute sore throat. Secondary measures included total doses of oral analgesics and time lost from work/activity.

\section{Methods}

We recruited Department of Defense beneficiaries at the Mike O'Callaghan Federal Medical Center who were $\geq 18$ years old, had a scheduled clinic visit for acute sore throat, and had a pain score of at least 5 on a 0 - to 10 -point visual analog scale. Acute sore throat was defined as "any patient presenting of their own volition to the clinic with a chief complaint of throat pain/sore throat/pharyngitis." Providers notified research assistants, who then approached potential subjects at the time of their appointment to consider enrollment in the study. Exclusion criteria were known pregnancy; absence of ear(s); active cellulitis of 1 or both ears; ear anatomy precluding the identification of acupuncture landmarks; non-English speaking; use of hearing aids precluding the insertion of needles; history of significant gastrointestinal bleeding; history of gastric bypass; known peritonsillar abscess (diagnosed at either a previous or the current visit); throat, mouth, or esophageal cancer; oral steroid use; chronic kidney disease (stage 2 or worse); concurrent use of pain medications for other conditions; inability to comply with study protocol; or allergy to study medications. The institutional review board at the Wilford Hall Ambulatory Surgical Center approved this study.

Following enrollment, research associates randomized subjects into 2 groups using a random number generator via block randomization (blocks of 4): group 1, standard treatment alone (STD group); group 2, standard treatment plus BFA (BFA group). Standard treatment was defined as oral nonsteroidal anti-inflammatory medication (600 $\mathrm{mg}$ ibuprofen) with or without oral antibiotics. All subjects were prescribed ibuprofen by their treating provider after review of their record for exclusion criteria. Decisions to treat with antibiotics were

Figure 1. Semipermanent ASP needle placement in battlefield acupuncture.

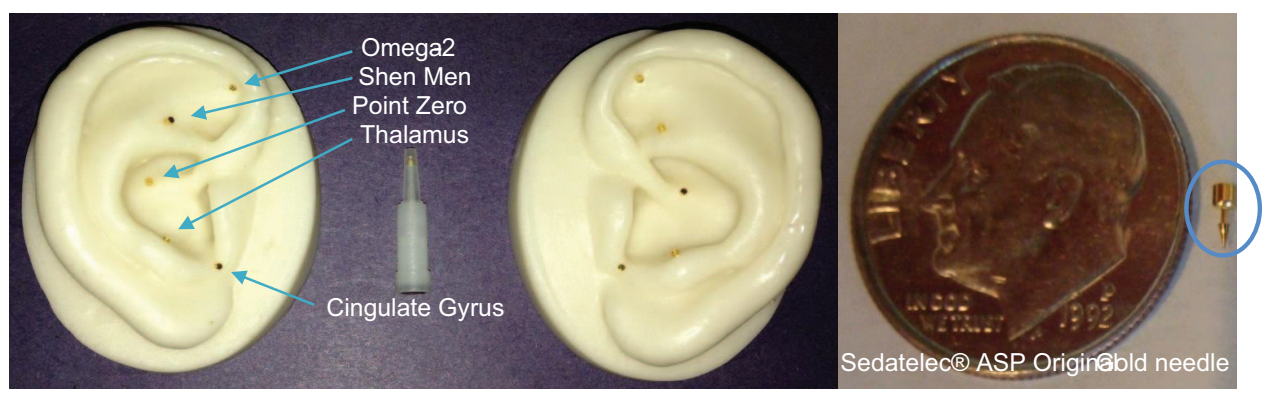


based on results of rapid streptococcus antigen testing; patients with positive results were prescribed either penicillin or another appropriate antibiotic (in the setting of penicillin allergy). We recruited 54 subjects, and each group included 27 subjects.

The study used Sedatelec ASP Original Gold needles $^{22}$ in the BFA group. These acupuncture needles are sterile, gold-plated, semipermanent ear acupuncture needles for single use (Figure 1). Subjects in the BFA group were given acupuncture treatment by physician investigators trained in the technique immediately after their visit with the treating provider, according to their randomization group. One investigator was a medical acupuncturist. All other investigators lacked formal acupuncture training with the exception of BFA. The right and left ears were cleansed with an alcohol swab before ear acupuncture. These subjects had semipermanent needles placed in some or all the following points on 1 or both ears, depending on their clinical response to the acupuncture: cingulate gyrus, thalamus, omega 2, point zero, and shenmen (in that order; Figure 1). Acupuncture treatment could consist of up to 10 needles, but if a subject reported resolution of pain, the acupuncture protocol was stopped. A credentialed medical acupuncturist provided intermittent and random "spot checks" of needle placement to ensure quality.

Unblinded research associates measured pain scores for both groups before any treatment and again for both groups 15 minutes after the initial pain score while they waited in the clinic. After discharge from the clinic, an unblinded research associate contacted the subjects via phone, E-mail or text message at 6,24 , and 48 hours. These contacts assessed the subject's pain level, cumulative doses of ibuprofen $600 \mathrm{mg}$ taken, and time missed from work/activities using a standard questionnaire developed for this protocol. For any patients who took other analgesics, naproxen $500 \mathrm{mg}$ and acetaminophen $1000 \mathrm{mg}$ were considered equal to ibuprofen $600 \mathrm{mg}$ for the purpose of calculating medication dosages.

ASP needles are designed to stay in the ear for several days, so we instructed subjects to allow the needles to fall out on their own, per usual practice throughout the world. We also taught subjects how to remove the needles if they became bothersome. We encouraged them to come into the clinic for removal or to remove them on their own if the needles had not fallen out within 1 week. There are no known reports in the literature of injury secondary to contact with an ASP needle that has fallen out of an ear.

We calculated the sample size before the study and determined that 27 subjects per group would detect a pain difference of 2 points, with a power 0.8 and an $\alpha$ of 0.05 . We used paired $t$ tests to compare outcomes of pain score, hours missed from work, and medication use. Because we used multiple univariate tests to investigate effects, the Bonferroni method was used post hoc to correct the level of significance for multiple comparisons; since we tested 3 hypotheses, we reduced the significant $\alpha$ to $0.017 .{ }^{23} \mathrm{We}$ planned on using intentto-treat analysis for any missing data and used the carry forward method to deal with any missing data. Statistics were calculated using Stata SE10 (StataCorp, College Station, TX).

\section{Results}

A total of 59 patients were approached to enroll in the study; 3 patients were prescribed steroids as part of the treatment for sore throat, so they were not enrolled, whereas 56 patients were enrolled in the study and randomized into 1 of the 2 groups. Two patients were lost to follow-up after randomization (Figure 2). Demographic data, including age and sex, were similar between the 2 groups (Table 1). Distribution of males and females between groups was similar; however, there were more women than men overall in the study (19 men vs 35 women). Use of antibiotics ( 2 in the STD group vs 3 in the BFA group) and return visits to the clinic ( 2 in the STD group vs 1 in the BFA group) were also similar between the 2 groups (Table 1). All subjects were prescribed ibuprofen per the protocol except for 2 patients in the STD group. One was prescribed naproxen and the other was prescribed acetaminophen per the treating physician's preference. These medications were treated the same as ibuprofen in the total dose calculations.

\section{Pain Scores}

Pain scores were similar at baseline between the 2 groups (STD group, 6.2 vs BFA group, 6.4; $P=$ $.52)$. Patients in the BFA group reported significantly less pain at 15 minutes (STD group, 6.0 vs BFA group, 2.6; $P<.0001$ ), 6 hours (STD group, 
Figure 2. Randomization and enrollment of study patients. BFA, battlefield acupuncture; STD, standard treatment.

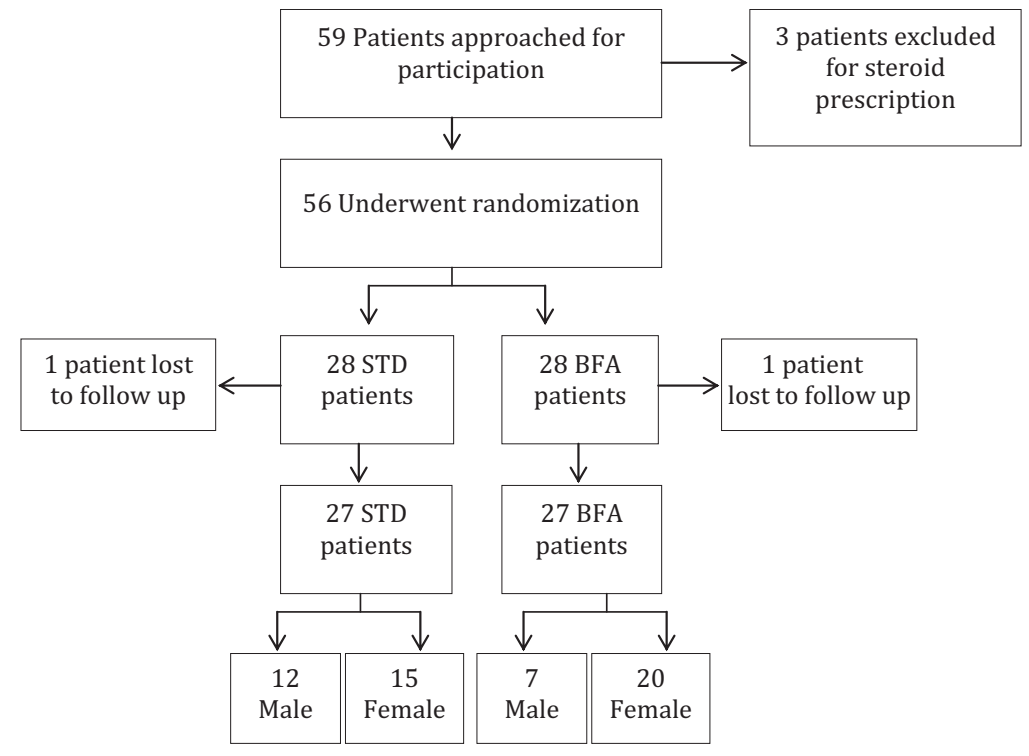

4.8 vs BFA group, 2.5; $P=.0005)$, and 24 hours (STD group, 4.1 vs BFA group, $1.9 ; P=.0006$ ). The difference in pain scores at 48 hours did not reach statistical significance (STD group, 2.3 vs BFA group, $1.3 ; P=.063$ ) (Table 2; Figure 3).

\section{Ibuprofen Usage}

Patients in the BFA group reported taking fewer cumulative doses of ibuprofen at 6 hours (STD group, 1.1 vs BFA group, $0.4 ; P=.003), 24$ hours (STD group, 2.6 vs BFA group, 1.4; $P=.004$ ), and 48 hours (STD group, 4.1 vs BFA group, 2.2; $P=$ 0.009) (Table 2; Figure 4).

\section{Missed Work/Activity Hours}

Any difference in time missed from work (hours) did not reach statistical significance (Table 2; Figure 5).

Figure 3. Mean pain scores of the standard treatment (STD) group versus the battlefield acupuncture (BFA) group.

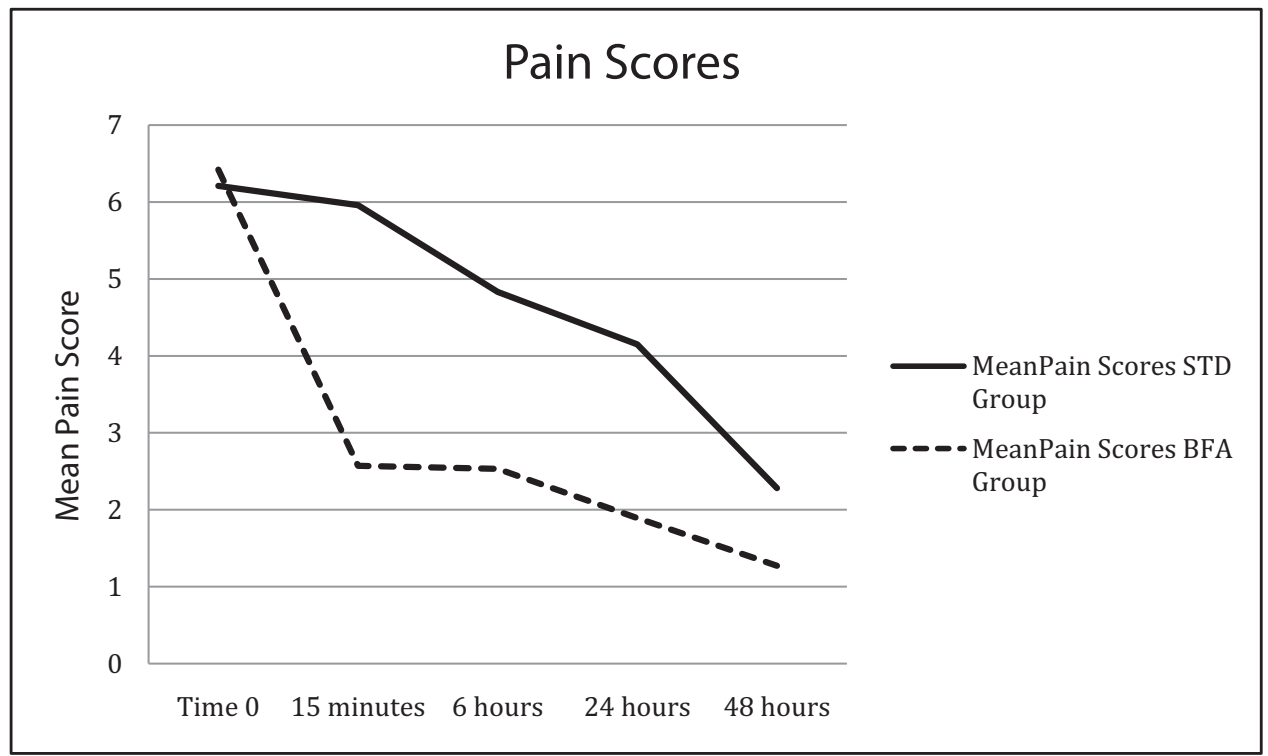


Figure 4. Mean doses of ibuprofen among the standard treatment (STD) group versus the battlefield acupuncture (BFA) group.

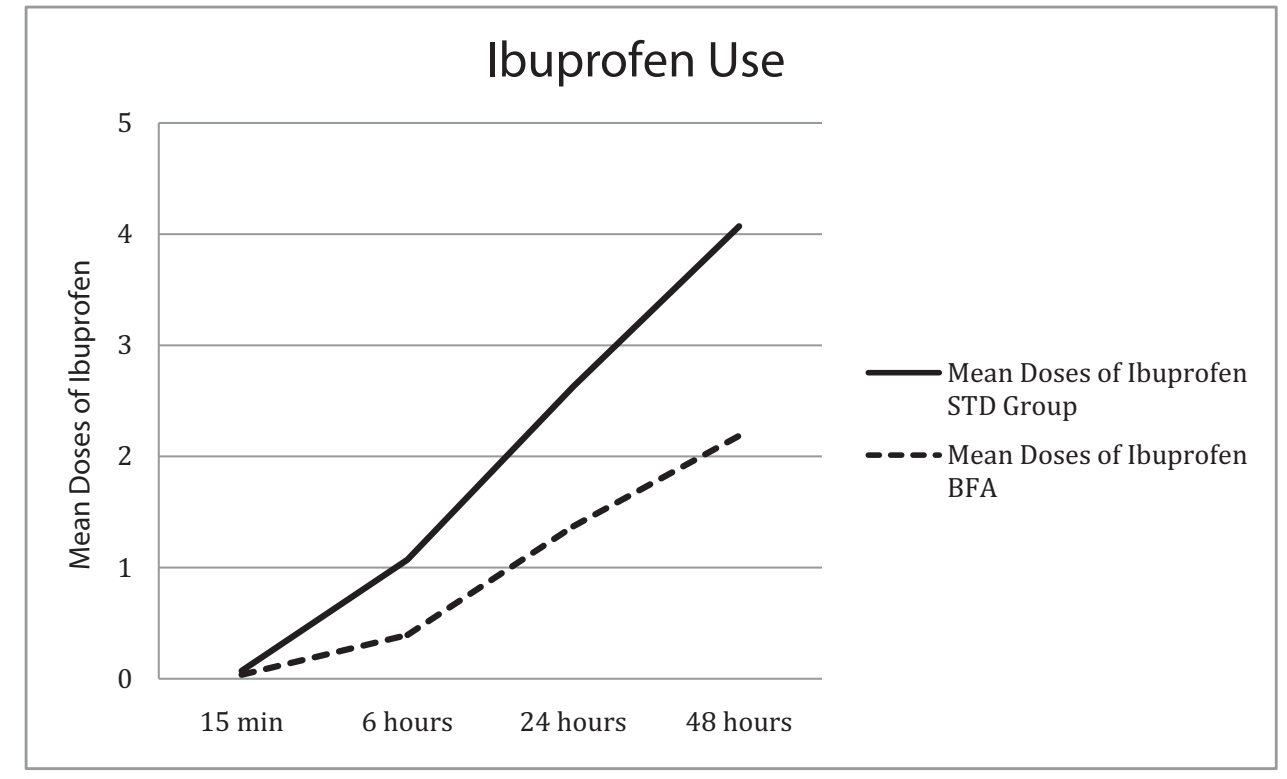

\section{Discussion}

Compared with usual treatment, BFA seems to reduce pain and decrease medication use for acute sore throat. However, it did not reduce time missed from work/activities among patients with acute sore throat. This study adds to the general body of

Figure 5. Missed work hours among the standard treatment (STD) group versus the battlefield acupuncture (BFA) group.

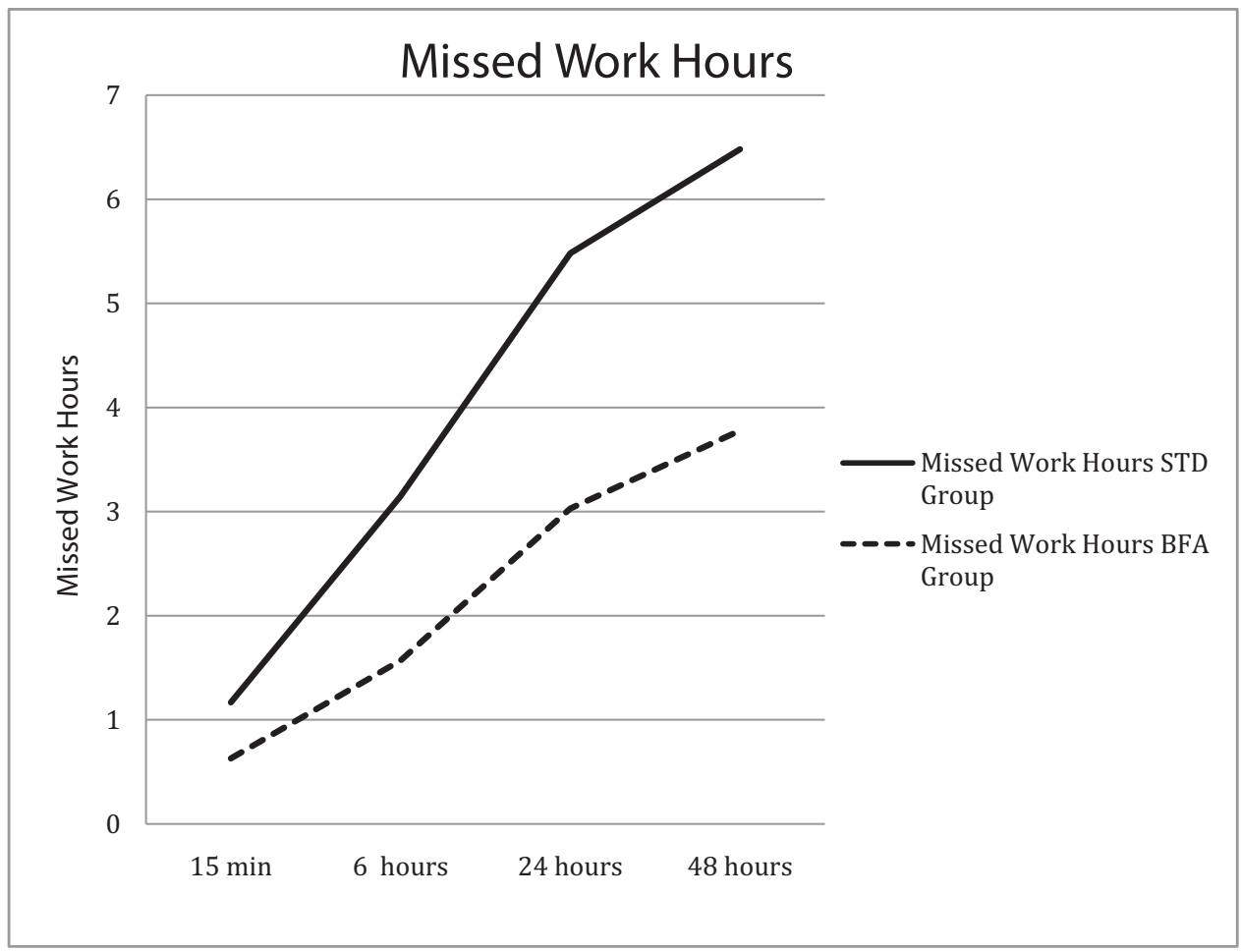


Table 1. Characteristics of Subjects

\begin{tabular}{lccc}
\hline & $\begin{array}{c}\text { Standard } \\
\text { Treatment } \\
\text { Group }\end{array}$ & $\begin{array}{c}\text { Battlefield } \\
\text { Acupuncture } \\
\text { Group }\end{array}$ & $\begin{array}{c}P \\
\text { Value }\end{array}$ \\
\hline Mean age (years) & 31 & 34 & .48 \\
Sex (n) & & & \\
$\quad$ Male & 12 & 7 & .25 \\
$\quad$ Female & 15 & 20 & .40 \\
Subjects using antibiotics (n) & 2 & 3 & .65 \\
Return clinic visit within 2 & 2 & 1 & .56 \\
$\quad$ weeks of initial & & & \\
$\quad$ encounter (n) & & & \\
\hline
\end{tabular}

evidence that auricular acupuncture is an efficacious treatment, and it specifically indicates that $\mathrm{BFA}$, as a protocol, may be effective at reducing pain from a common cause in the outpatient clinic.

For acute outpatient complaints that are not life threatening, there is probably no more important or common quality of life indicator than pain. Our study demonstrates a statistically and clinically significant reduction in pain using BFA to treat sore throat for up to 24 hours, and trended toward significance at 48 hours. Pain scores in both groups were low at 48 hours, probably because of the self-limiting nature of most causes of sore throat. Prolonged pain relief with acupuncture is beneficial for multiple reasons, and future research may include pain syndromes with a longer natural history, such as physical trauma or postsurgical pain.

Reduction in medication usage is also a surrogate for true pain relief and decreases overall cost. In addition, pain medications such as NSAIDs have potential side effects that can be avoided with alternative pain relief modalities. Complications of NSAIDs were not followed in this study because NSAID use within 1 week of initiation in healthy patients rarely results in gastrointestinal bleeding, ${ }^{24}$ and measuring kidney function for routine prescription of NSAIDs is not standard practice. Any potential difference in complications caused by a reduction in medication use between groups would be almost impossible to demonstrate since the study was powered to detect efficacy, not medication complications. We did observe an approximately $50 \%$ reduction in medication doses at 24 and 48 hours (Table 2). Though it is difficult to determine whether this is clinically relevant, if tested in other pain syndromes, there is a potential for BFA to reduce morbidity from prolonged NSAID exposure.

The ability to return to work sooner is an important indicator of true pain relief and overall well-being, and it is an outcome that matters to

Table 2. Pain Scores, Ibuprofen Doses, and Missed Work Hours Among the Standard Treatment Group versus the Battlefield Acupuncture Group

\begin{tabular}{|c|c|c|c|}
\hline & $\begin{array}{l}\text { Standard Treatment } \\
(95 \% \mathrm{CI})\end{array}$ & $\begin{array}{c}\text { BFA }+ \text { Standard Treatment } \\
(95 \% \mathrm{CI})\end{array}$ & $P$ Value ${ }^{*}$ \\
\hline \multicolumn{4}{|c|}{ Pain scores $(0-10)$} \\
\hline 0 minutes & $6.2(5.7-6.7)$ & $6.4(6.0-6.8)$ & .52 \\
\hline 15 minutes & $6.0(5.4-6.6)$ & $2.6(1.7-3.5)$ & $<.0001$ \\
\hline 6 hours & $4.8(4.0-5.6)$ & $2.5(1.6-3.4)$ & .0005 \\
\hline 24 hours & $4.1(3.3-4.9)$ & $1.9(1.0-2.8)$ & .0006 \\
\hline 48 hours & $2.3(1.6-3.0)$ & $1.3(0.6-2.0)$ & .063 \\
\hline \multicolumn{4}{|c|}{ Ibuprofen doses taken (n) } \\
\hline 15 minutes & $0.07(0-0.08)$ & $0.04(0-0.012)$ & .56 \\
\hline 6 hours & $1.07(0.69-1.45)$ & $0.39(0.2-0.58)$ & .003 \\
\hline 24 hours & $2.63(1.95-3.31)$ & $1.37(0.92-1.82)$ & .004 \\
\hline 48 hours & $4.07(2.9-5.24)$ & $2.19(1.44-2.94)$ & .009 \\
\hline \multicolumn{4}{|c|}{ Missed work hours } \\
\hline 15 minutes & $1.13(0.49-1.77)$ & $0.61(0-1.25)$ & .25 \\
\hline 6 hours & $3.19(1.91-4.47)$ & $1.57(0.66-2.48)$ & .05 \\
\hline 24 hours & $5.48(3.25-7.71)$ & $3.04(1.34-4.74)$ & .09 \\
\hline 48 hours & $6.48(3.99-8.97)$ & $3.78(1.63-5.93)$ & .11 \\
\hline
\end{tabular}

*Pairwise comparisons by $t$ test.

BFA, battlefield acupuncture; CI, confidence interval. 
both clinicians and patients. Lost work hours also add to the overall cost of illness. Because of the short follow-up period in this study, we chose to measure hours missed from work rather than days. We also felt this was an appropriate secondary measure considering that the US Department of Labor reports that in 2012 there were 75.3 million workers who were paid at hourly rates, which would make hours, in addition to days, of lost work important to these workers. ${ }^{25}$ Our study demonstrated only trends toward reduced work missed in the BFA group and did not demonstrate any statistical difference in missed work hours between the 2 groups. We also found that the standard deviations for missed work hours were large, indicating a wide variation in missed time between individuals. We did not power this study to accurately measure this secondary outcome, and a larger sample size would likely demonstrate whether there truly is no difference.

Although the study was adequately powered, the sample size in this study is small. Randomization helps to mitigate the probability that results are due to chance; however, the risk of group imbalances does increase with smaller sample sizes. Symptom duration at presentation was not recorded in our study, which could have confounded results if one of the groups tended to present later or earlier in the course of illness; however, we feel that such variation between the groups is unlikely.

Neither acupuncturists, patients, nor data collectors were blinded in this study. While it would have been possible to blind the data collector, this is a limitation that was unavoidable because of budget and personnel limitations.

More women than men participated in our study. We are unable to explain the cause of this imbalance, except that it is likely in this military population that men were less likely to present for health complaints. The current literature suggests little difference in the willingness of men versus women to access the civilian health care system, ${ }^{26,27}$ but this may differ among a military population. Another possible explanation is that some jobs within the military setting would prohibit the use of ASP needles for safety reasons, that is, prohibitive headgear or the risk of having loose objects near the flight line. Some studies also suggest that women are more likely to try integrative therapies such as acupuncture. ${ }^{28}$ Similar proportions of men and women were present in each group.
There was no "sham" group in our study. Sham refers to placing needles in nonacupuncture points as a purported control for the sensation of having needles placed in the ear. Prior studies have compared acupuncture with sham acupuncture, but very few compare acupuncture to standard treatment. We decided to directly compare BFA to standard treatment. First, there is evidence that even sham acupuncture can cause analgesia by activating afferent, nonmyelinated "C" fibers, so sham may actually be a treatment, not just a control for a placebo effect. ${ }^{29}$ In addition, we wanted to perform an effectiveness rather than an efficacy trial. We believe that this approach is more pragmatic and "reflects the complexity and diversity of actual clinical practice." ${ }^{30}$ Demonstrating a nearly $50 \%$ reduction in pain scores along with reduction in medication usage longitudinally provides clinically useful information.

The procedure of performing BFA takes 3 to 10 minutes, and 10 ASP needles cost approximately $\$ 5$. Physicians treating patients with acute sore throat can easily justify an evaluation and management code of 99212 to 99214 . In addition to this evaluation and management code, the Current Procedural Terminology code for acupuncture is 97810 and is worth 0.60 relative value units. Many insurance carriers pay for acupuncture. Thus, performing acupuncture within a clinic visit can be financially rewarding.

Variation in the technique, training, and experience of acupuncturists makes studying acupuncture difficult. Location of acupuncture points, depth to which needles are inserted, and manipulation of needles with manual or electric stimulation are just a few examples of potentially confounding factors. In addition, it is difficult to control for the intangible effects of time spent with a patient, placing needles in the body, and physical contact. This study mitigates these confounding factors in several ways. BFA uses 5 specific points on each ear in a systematic protocol that leaves little room for misinterpretation. There is no manipulation of the needles, and the depth of insertion is standardized because of the construction of the semipermanent needles.

BFA is a simple protocol that can be learned in a single 4-hour session by nonacupuncturists. ${ }^{31}$ The procedure does not require specific follow-up. The body expels the semipermanent needles as skin re-epithelializes over several days, or needles can be 
removed by the patient. By contrast, traditional acupuncture requires months to years of training (typically 300 hours of training for physicians and 4 years of training for licensed acupuncturists). The practice of traditional acupuncture involves subtle variation in techniques that can be both time consuming and difficult to learn. In addition, a single traditional acupuncture session can last from 30 minutes to over 1 hour. Providers using BFA do not learn the subtleties of acupuncture, but they do learn a limited, time-efficient adjunct for patients with pain. A nonacupuncturist can easily address conventional medical problems and still provide BFA for acute pain during a short appointment.

The possibilities for future research are extensive and include acute pain syndromes-both organic and iatrogenic-encountered in an outpatient or inpatient setting. Other relevant questions worth exploring include the willingness of nonacupuncturists to incorporate a technique like BFA into their clinical practice, the willingness of patients to use this and other acupuncture treatments, and the quality of BFA performed by nonacupuncturists.

Treating acute sore throat with BFA could allow physicians to reduce pain scores for up to 24 hours when compared with conventional therapy. BFA also seems to reduce medication usage, and future research may determine whether it can aid patients in returning to normal activities sooner. As a technique easily learned by nonacupuncturists, BFA may be a valuable treatment option for sore throat pain.

The following individuals were instrumental in assisting with patient recruitment, acupuncture treatment, patient follow-up, data collection, and data analysis: Jill M. Clark, MBA/HCM, CCRP, CCRC; Tracy Bogdanovich, CCRC; Aaron Barnett, BA; Jessica Johnson, MPH, CHES; Kirk Middleton, DO; Eric Abbott, DO; Heather Pickett, DO; Pam Driggers, PhD, MPH; Anthony Viera, MD. The views expressed in this article are those of the authors and do not reflect the official policy or position of the U.S. Air Force, the Department of Defense, or the U.S. Government.

\section{References}

1. Centers for Disease Control and Prevention. National Ambulatory Medical Care Survey: 2009 summary tables. Available from: http://www.cdc.gov/nchs/data/ ahcd/namcs_summary/2009_namcs_web_tables.pdf. Accessed September 22, 2015.

2. Shulman ST, Bisno AL, Clegg HW, et al. Clinical practice guideline for the diagnosis and management of group A streptococcal pharyngitis: 2012 update by the Infectious Diseases Society of America. Clin Infect Dis 2012;55:e86-102.

3. Shy BD, Strayer RJ. Nonsteroidal anti-inflammatory drugs are an effective alternative to corticosteroids to treat pain in pharyngitis. Ann Emerg Med 2014;64: 686.

4. Russo M, Bloch M, de Looze F, Morris C, Shephard A. Flurbiprofen microgranules for relief of sore throat: a randomised, double-blind trial. Br J Gen Pract 2013;63:e149-55.

5. Centers for Disease Control and Prevention. Get smart: know when antibiotics work. Available from: http://www.cdc.gov/getsmart/antibioticuse/symptom-relief.html. Accessed January 8, 2015.

6. Clement-Jones V, McLoughlin L, Tomlin S, Besser GM, Rees LH, Wen HL. Increased $\beta$-endorphin but not Met-enkephalin levels in human cerebrospinal fluid after acupuncture for recurrent pain. Lancet 1980;316:946-9.

7. Soliman N, Frank BL. Auricular acupuncture and auricular medicine. Phys Med Rehabil Clin N Am 1999; 10:547-54.

8. Cho ZH, Fallon J, Wong EK. Neuro-Acupuncture. Vol I: Basic Neuroscience. Los Angeles: Q-Puncture Inc.; 2001.

9. Niemtzow R. Battlefield acupunture. Med Acup 2007;19:225-8.

10. Nogier P. Treatise of auriculotherapy. Moulins-lesMetz, Maisonneuve, France; 1972.

11. Plunkett A, Turabi A, Wilkinson I. Battlefield analgesia: a brief review of current trends and concepts in the treatment of pain in US military casualties from the conflicts in Iraq and Afghanistan. Pain Manag 2012;2:231-8.

12. Usichenko TI, Dinse M, Lysenyuk VP, Wendt M, Pavlovic D, Lehmann C. Auricular acupuncture reduces intraoperative fentanyl requirement during hip arthroplasty-a randomized double blinded study. Acupunct Electrother Res 2006;31:213-21.

13. Usichenko TI, Kuchling S, Witstruck T, et al. Auricular acupuncture for pain relief after ambulatory knee surgery: a randomized trial. CMAJ 2007;176: $179-83$.

14. Usichenko TI, Lehmann Ch, Ernst E. Auricular acupuncture for postoperative pain control: a systematic review of randomised clinical trials. Anaesthesia 2008;63:1343-8.

15. Wang SM, Dezinno P, Lin EC, et al. Auricular acupuncture as a treatment for pregnant women who have low back and posterior pelvic pain: a pilot study. Am J Obstet Gynecol 2009;201:271.e1-9.

16. Allais G, Romoli M, Rolando S, Castagnoli Gabellari I, Benedetto C. Ear acupuncture in unilateral migraine pain. Neurol Sci 2010;31(Suppl 1):S185-7.

17. Goertz CM, Niemtzow R, Burns SM, Fritts MJ, Crawford CC, Jonas WB. Auricular acupuncture in 
the treatment of acute pain syndromes: a pilot study. Mil Med 2006;171:1010-4.

18. Hunter RF, McDonough SM, Bradbury I, et al. Exercise and auricular acupuncture for chronic lowback pain: a feasibility randomized-controlled trial. Clin J Pain 2012;28:259-67.

19. Sator-Katzenschlager SM, Wölfler MM, KozekLangenecker SA, et al. Auricular electro-acupuncture as an additional perioperative analgesic method during oocyte aspiration in IVF treatment. Hum Reprod 2006;21:2114-20.

20. Lewis SM, Clelland JA, Knowles CJ, Jackson JR, Dimick AR. Effects of auricular acupuncture-like transcutaneous electric nerve stimulation on pain levels following wound care in patients with burns: a pilot study. J Burn Care Rehab 1990;11:322-9.

21. Yeh CH, Chiang YC, Hoffman SL, et al. Efficacy of auricular therapy for pain management: a systematic review and meta-analysis. Evid Based Complement Alternat Med 2014;2014:934670. doi:10.1155/2014/ 934670.

22. Lhasa OMS. ASP needles. Available from: http:// www.lhasaoms.com/ASP-Ear-Needles.html. Accessed December 3, 2014.

23. Abdi H. Bonferroni and Sidák corrections for multiple comparisons. In N.J. Salkind (ed.). Encyclopedia of Measurement and Statistics. Vol. 1. Thousand Oaks, CA: Sage; 2007.

24. Langman MJ, Weil J, Wainwright P, et al. Risks of bleeding peptic ulcer associated with individual non- steroidal anti-inflammatory drugs. Lancet 1994;343: 1075-8.

25. United States Department of Labor, Bureau of Labor Statistics. Minimum wage workers account for 4.7 percent of hourly paid workers in 2012. TED: The Economics Daily. Available from: http://www.bls.gov/opub/ted/ 2013/ted_20130325.htm. Accessed April 13, 2015.

26. Wang Y, Freemantle N, Nazareth I, Hunt K. Gender differences in survival and the use of primary care prior to diagnosis of three cancers: an analysis of routinely collected UK general practice data. PLoS One 2014;9:e101562.

27. Hunt K, Adamson J, Hewitt C, Nazareth I. Do women consult more than men? A review of gender and consultation for back pain and headache. J Health Serv Res Policy 2011;16:108-17.

28. Bishop L, Lewith G. Who uses CAM? A narrative review of demographic characteristics and health factors associated with CAM use. Evid Based Complement Alternat Med 2010;7:11-28.

29. Lund I, Lundeberg T. Are minimal, superficial or sham acupuncture procedures acceptable as inert placebo controls? Acupunct Med 2006;24:13-5.

30. Ware JH, Hamel MB. Pragmatic trials-guides to better care? N Engl J Med 2011;364:1685-7.

31. Leggit JC. Introduction of integrative health and acupuncture to pre-clerkship medical students. Med Acupunct 2014;26:226-9. 